\title{
DMRG study of exciton condensation in the extended Falicov-Kimball model
}

\author{
P. Farkašovský \\ Institute of Experimental Physics, Slovak Academy of Sciences, Watsonova 47, 04353 Košice, Slovakia
}

Received May 15, 2020, in final form August 13, 2020

\begin{abstract}
The formation and condensation of excitonic bound states of conduction-band electrons and valence-band holes surely belongs to one of the most exciting ideas of contemporary solid state physics. In this short review we present the latest progress in this field reached by the density-matrix-renormalization-group (DMRG) calculations within various extensions of the Falicov-Kimball model. Particular attention is paid to a description of crucial mechanisms (interactions) that affect the stability of the excitonic phase, and namely: (i) the interband $d$ - $f$ Coulomb interaction, (ii) the $f$-electron hopping, (iii) the nonlocal hybridization with odd and even parity, (iv) combined effects of the local and nonlocal hybridization, (v) the nearest-neighbor Coulomb interaction between $d$ and $f$ electrons and (vi) the correlated hopping. The relevance of numerical results obtained within different extensions of the Falicov-Kimball model for a description of the real $d$ - $f$ materials is widely discussed.
\end{abstract}

Key words: Falicov-Kimball model, quantum condensates, one-dimensional systems

\section{Introduction}

The formation of excitonic quantum condensates is an intensively studied continuous problem in condensed matter physics [1-4]. Whilst theoretically predicted a long time ago [5], no conclusive experimental proof of the existence of the excitonic condensation has been achieved yet. However, the latest experimental studies of materials with strong electronic correlations showed that promising candidates for the experimental verification of the excitonic condensation could be $\mathrm{TmSe}_{0.45} \mathrm{Te}_{0.55}$ [6, 7], $1 T-\mathrm{TiSe}_{2}$ [8-11], $\mathrm{Ta}_{2} \mathrm{NiSe}_{5}$ [12], or a double bilayer graphene system [13]. In this regard, the mixed valence compound $\mathrm{TmSe}_{0.45} \mathrm{Te}_{0.55}$ was argued to exhibit a pressure-induced excitonic instability, related to an anomalous increase in the electrical resistivity [6, 7]. In particular, detailed studies of the pressureinduced semiconductor-semimetal transition in this material [based on the Hall effect, electrical and thermal (transport) measurements] showed that excitons are created in a large quantity and condense below $20 \mathrm{~K}$. On the other hand, in the layered transition-metal dichalcogenide $1 T-\mathrm{TiSe}_{2}$, a BCS-like electronhole pairing was considered as the driving force for the periodic lattice distorsion [8-11]. Moreover, quite recently, the excitonic-insulator state was probed by angle-resolved photoelectron spectroscopy in the semiconducting $\mathrm{Ta}_{2} \mathrm{NiSe}_{5}$ compound [12]. These results have stimulated further experimental and theoretical studies with regard to the formation and possible condensation of excitonic bound states of electron and holes in correlated systems. At present, it is generally accepted that the minimal theoretical model for a description of excitonic correlations in these materials could be the FalicovKimball model [14] and its extensions which were successfully used in the past years to test the exciting idea of electronic ferroelectricity [15-25] that is directly related with the formation of an excitonic insulator [26-35]. In its original form, the Falicov-Kimball model describes a two-band system of localized $f$ electrons and itinerant $d$ electrons with short-ranged $f$ - $d$ Coulomb interaction $U$ :

$$
H_{0}=\sum_{i j} t_{i j} d_{i}^{+} d_{j}+U \sum_{i} f_{i}^{+} f_{i} d_{i}^{+} d_{i}+E_{f} \sum_{i} f_{i}^{+} f_{i}
$$


where $f_{i}^{+}, f_{i}$ are the creation and annihilation operators for an electron in the localized state at lattice site $i$ with the binding energy $E_{f}$ and $d_{i}^{+}, d_{i}$ are the creation and annihilation operators of the itinerant spinless electrons in the $d$-band Wannier state at site $i$.

The first term of (1.1) is the kinetic energy corresponding to quantum-mechanical hopping of the itinerant $d$ electrons between sites $i$ and $j$. These intersite hopping transitions are described by the matrix elements $t_{i j}$, which are $-t_{d}$ if $i$ and $j$ are the nearest neighbors and zero otherwise (in what follows all parameters are measured in units of $t_{d}$ ). The second term represents the on-site Coulomb interaction between the $d$-band electrons with density $n_{d}=\frac{1}{L} \sum_{i} d_{i}^{+} d_{i}$ and the localized $f$ electrons with density $n_{f}=\frac{1}{L} \sum_{i} f_{i}^{+} f_{i}$, where $L$ is the number of lattice sites. The third term stands for the localized $f$ electrons whose sharp energy level is $E_{f}$.

Since in this simple model, the local occupation number $f_{i}^{+} f_{i}$ commutates with the total Hamiltonian of the system, the local $f$-electron number is a strictly conserved quantity and thus the $d$ - $f$ electron coherence cannot be established in such a system. If hybridization $H_{V}=V \sum_{i} d_{i}^{+} f_{i}+f_{i}^{+} d_{i}$ between both bands is included, the $f$ charge occupation is no longer a good quantum number, and it is possible to build coherence between $d$ and $f$ electrons. Hybridization between the itinerant $d$ and localized $f$ states, however, is not the only way to develop $d-f$ coherence. Theoretical works of Batista et al. [22, 23] showed that the ground state with a spontaneous electric polarization can also be induced by the nearest-neighbor $f$-electron hopping $H_{t_{f}}=-t_{f} \sum_{<i, j>} f_{i}^{+} f_{j}$, but only for dimensions $D>1$. In the strong coupling limit, this result was proven by mapping the extended Falicov-Kimball model into the $x x z$ spin $1 / 2$ model with a magnetic field along the $z$-direction, while in the intermediate coupling regime the ferroelectric state was identified numerically by constrained path Monte Carlo (CPMC) technique. Based on these results, the authors postulated the following conditions that favour the formation of the electronically driven ferroelectric state: (a) The system must be in a mixed-valence regime and the two bands involved must have different parity. (b) It is best, though not necessary, if both bands have similar bandwidths. (c) A local Coulomb repulsion $(U)$ between the different orbitals is required.

Later on this model was extensively used to describe different phases in the ground state and special properties of the excitonic phase [26-32]. It was found that the ground state phase diagram exhibits a very simple structure consisting of only four phases, and namely, the full $d$ and $f$ band insulator (BI), the excitonic insulator (EI), the charge-density-wave (CDW) and the staggered orbital order (SOO). The EI is characterized by a nonvanishing $\left\langle d^{+} f\right\rangle$ average. The CDW is described by a periodic modulation in the total electron density of both $f$ and $d$ electrons, and the SOO is characterized by a periodic modulation in the difference between the $f$ and $d$ electron densities.

In this article we focus our attention on the properties of the EI phase induced by local hybridization $V$ in the one dimension. Although it is generally known that there is no nonvanishing $P_{d f}=\left\langle d^{+} f\right\rangle$ expectation value in the limit of vanishing hybridization (no spontaneous hybridization), the studies that we performed in the past years on various extensions of the original Falicov-Kimball model showed that it is possible to dramatically enhance excitonic correlations in the limit of small, but finite $V$ by additional interactions/factors [36-39]. The effects of most important interactions are discussed in this review. In particular, there are: (i) the interband $d$ - $f$ Coulomb interaction, (ii) the $f$-electron hopping, (iii) the nonlocal hybridization with odd and even parity, (iv) combined effects of the local and nonlocal hybridization, ( $\mathrm{v}$ ) the nearest-neighbor Coulomb interaction between $d$ and $f$ electrons and (vi) the correlated hopping. The main goal of this review is not to examine the possibilities of spontaneous symmetry breaking (a spontaneous hybridization) in various extensions of the Falicov-Kimball model, but to show how these extensions (different interaction terms) influence the properties of the excitonic phase induced by local hybridization. All presented results were obtained within the density-matrixrenormalization-group (DMRG) method. where we typically keep up to 500 states per block, although in the numerically more difficult cases (where the DMRG results converge slower), we keep up to 1000 states. Truncation errors [40], given by the sum of the density matrix eigenvalues of the discarded states, vary from $10^{-6}$ in the worse cases to zero in the best cases. 

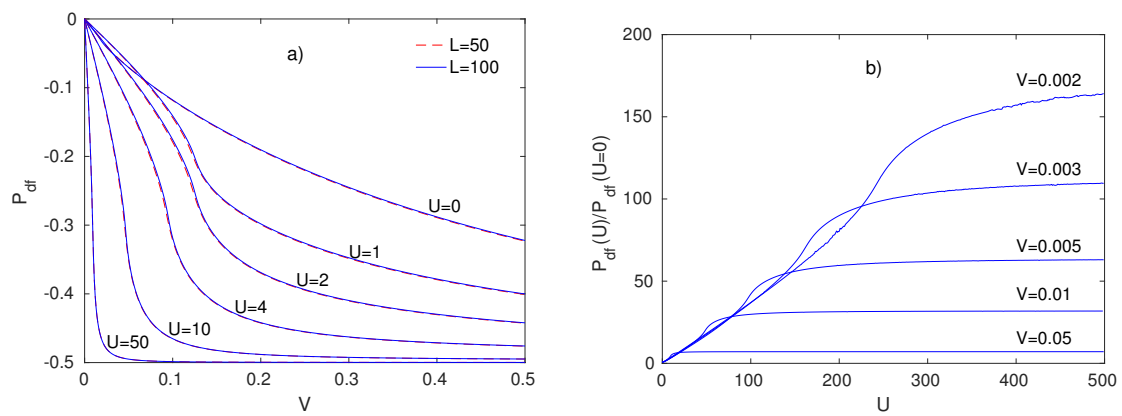

Figure 1. (Colour online) a) The hybridization dependence of the $d$-f-excitonic average $P_{d f}=\left\langle d_{i}^{+} f_{i}\right\rangle$ in the extended Falicov-Kimball model calculated for six different values of $U$ and two different values of $L$. The symmetric case $E_{f}=0$. b) The ratio of the interacting $P_{d f}(U)$ and non-interacting $P_{d f}(U=0)$ excitonic average as a function of $U$ calculated for several selected values of local hybridization $V$ on the cluster of $L=100$ sites [36].

\section{Results and Discussion}

\subsection{Effects of interband Coulomb interaction}

Let us start our review with the discussion of effects of the Coulomb interactions [36]. In this case, Hamiltonian consists of two terms: $H_{0}$, which is given by $(1.1)$ and $H_{V}=V \sum_{i} d_{i}^{+} f_{i}+f_{i}^{+} d_{i}$. Our DMRG results obtained for the symmetric case $E_{f}=0$ are summarized in figure 1 a and in figure $1 \mathrm{~b}$ where the $P_{d f}=\left\langle d_{i}^{+} f_{i}\right\rangle$ expectation value is shown as a function of hybridization for several values of Coulomb interaction $U$ (figure 1 a) and the ratio $\Delta=P_{d f}(U) / P_{d f}(U=0)$ for several values of $V$ (figure $1 \mathrm{~b}$ ). Figure 1 a clearly demonstrates that there is no nonvanishing $\left\langle d^{+} f\right\rangle$-expectation value in the limit of vanishing hybridization for all examined values of $U$. At the same time, these data reveal an important feature of the model and namely that the $P_{d f}$ expectation value is dramatically enhanced with increasing $U$ in comparison to the noninteracting case. This is explicitly shown in figure $1 \mathrm{~b}$ where the ratio of the interacting $P_{d f}(U)$ and non-interacting $P_{d f}(U=0)$ excitonic average is plotted for several selected values of hybridization. For all examined values of $\mathrm{V}$, the ratio $\Delta=P_{d f}(U) / P_{d f}(U=0)$ rapidly increases with increasing interband Coulomb interaction $U$ from its initial value $\Delta=1$ to its saturated value $\Delta=\Delta_{s}$ that also dramatically increases with a decreasing $V$. Indeed, while $\Delta_{s} \sim 7$ for $V=0.05$ its value increases up to $\sim 200$ for $V=0.002$. This result is very important from the point of view of real rare-earth materials with $d$ and $f$ electrons. In these materials the local hybridization is usually forbidden due to the crystal symmetry an thus the $d-f$ coherence cannot be established. However, according to our results, any infinitesimal hybridization, induced by some additional mechanism, could lead to a robust excitonic average due to the interband Coulomb interaction. Such an additional mechanism could be, for example, the electron-phonon interaction $H_{\mathrm{el}-\mathrm{ph}}$ that can be reduced to the phonon-mediated local hybridization (electron-electron interactions) by the standard canonical transformation of the form $\mathrm{e}^{S} \mathrm{He}^{-S}$, where the operator $S$ is determined so that $H_{\mathrm{el}-\mathrm{ph}}=-\left[S, H_{\mathrm{loc}}\right]$ and $H_{\mathrm{loc}}$ are all local terms corresponding to $f, d$ electrons and phonons [41].

To examine the nature of the EI state more in detail, we have calculated, in accordance with [31] and [32], the exciton-exciton correlation function $\left\langle b_{i}^{+} b_{j}\right\rangle$ with $b_{i}^{+}=d_{i}^{+} f_{i}$ and the excitonic momentum distribution $N(q)=\left\langle b_{q}^{+} b_{q}\right\rangle$ with $b_{q}^{+}=(1 / \sqrt{L}) \sum_{k} d_{k+q}^{+} f_{k}$. We have found that the exciton-exciton correlation function $\left\langle b_{i}^{+} b_{j}\right\rangle$ exhibits power-low correlations $|i-j|^{-\alpha}$ (with $\alpha$ between 3 and 4 ) and the excitonic momentum distribution $N(q)$ diverges for $q=0$ (see figure 2 a), signalizing a Bose-Einstein condensation of preformed excitons. Moreover, figure $2 \mathrm{~b}$ shows that the density of zero momentum excitons $n_{0}=\frac{1}{L} N(q=0)$ as well as the total exciton density $n_{T}=\frac{1}{L} \sum_{q} N(q)$ strongly depend on the values of the Coulomb interaction $U$ and that already for relatively small values of $U(U \sim 4)$ practically all particles are paired in electron-hole pairs with significant fraction of $n_{0} / n_{T} \sim 0.5$ excitons in the zero-momentum state. 

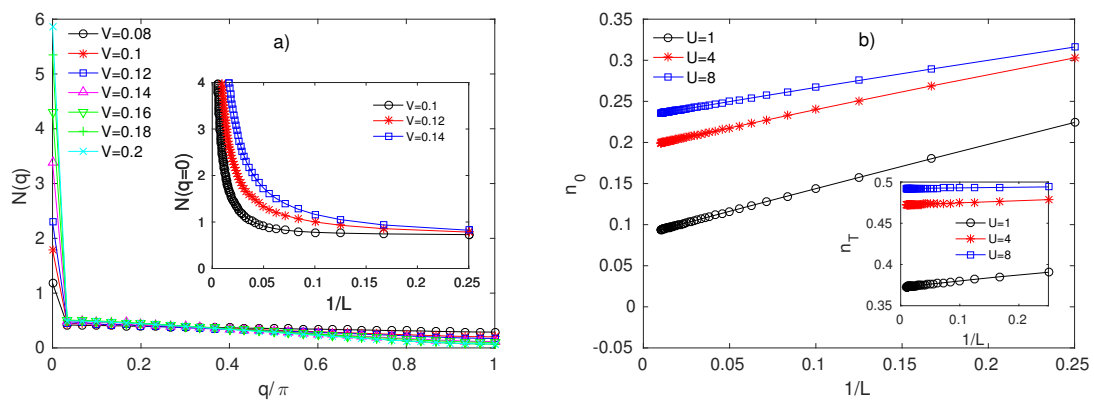

Figure 2. (Colour online) a) The excitonic momentum distribution $N(q)$ calculated for different values of $V$ at $U=1, E_{f}=0$ and $L=60$. The inset shows a divergence of $N(q=0)$ for $L \rightarrow \infty$ for three selected values of $V$. b) The density of zero momentum excitons $n_{0}$ and the total exciton density $n_{T}$ as functions of $1 / L$ calculated for several different values of $U$ at $V=0.2$ and $E_{f}=0$ [36].

\subsection{Effects of $f$-electron hopping}

With regard to the situation in real materials, where there always exists a finite overlap of $f$ orbitals on the neighbouring sites, it is interesting to ask what happens if the $f$-electron hopping $H_{t_{f}}=-t_{f} \sum_{<i, j>} f_{i}^{+} f_{j}$ is also taken into account [37]. In accordance with some previous theoretical studies, which documented strong effects of the parity of $f$ band on the stability of the excitonic phase [22, 23], we have examined the model for both the positive (the even parity) and negative (the odd parity) values of the $f$-electron hopping integrals $t_{f}$. The results of our non-zero $t_{f}$ DMRG calculations for $n_{0}$ are displayed in figure 3 and they clearly demonstrate that the zero-momentum condensate is suppressed in the limit of positive values of $t_{f}$, while it remains robust for negative values of $t_{f}$. This result
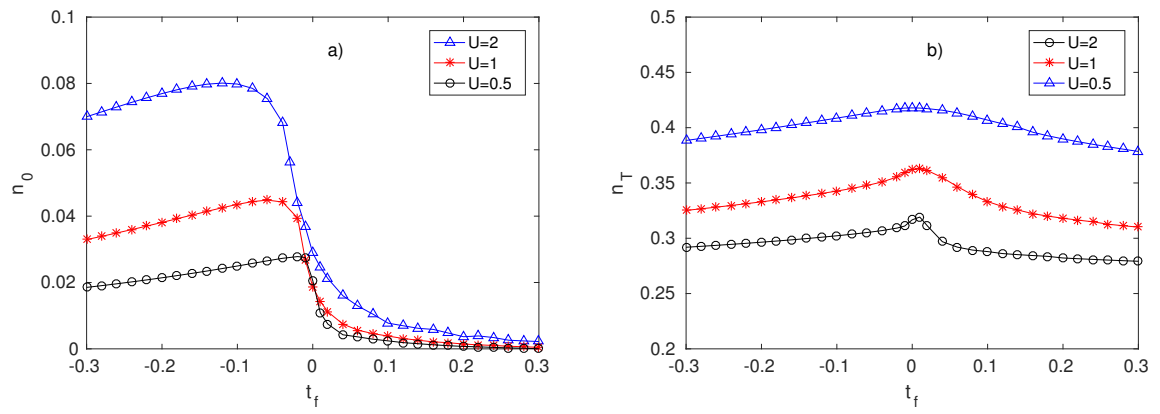

Figure 3. (Colour online) $n_{0}$ (a) and $n_{T}$ (b) as functions of $t_{f}$ calculated for three different values of $U$ $\left(E_{f}=0, V=0.1, L=\infty\right)$ [37].

is intuitively expected since our previous Hartree-Fock (HF) results [24] showed that only the negative values of $t_{f}$ stabilize the ferroelectric phase, while the positive values stabilize the antiferroelectric phase. The effect of $t_{f}$ is especially strong for $U$ small (see figure $3 \mathrm{a}$ ), where continuous but very steep changes of $n_{0}$ are observed for $t_{f} \rightarrow 0^{+}$. On the contrary, the total exciton density $n_{T}$ (figure $3 \mathrm{~b}$ ) exhibits only a weak dependence on the $f$-electron hopping parameter $t_{f}$, over the whole interval of $t_{f}$ values.

\subsection{Effects of $f$-level position (pressure)}

So far we have presented the results exclusively for $E_{f}=0$. Let us now briefly discuss the effect of the change of the $f$-level position [37]. This study is also interesting from the point of view that taking into account the parametrization between the external pressure and the position of the $f$ level $\left(E_{f} \sim p\right)$, one can also deduce, at least qualitatively, their $p$ dependences from the $E_{f}$ dependences of the ground 
state characteristics [42]. The resultant $E_{f}$ dependences of the density of zero momentum excitons $n_{0}$ are shown in figure 4 a for several values of $V$ and $U=0.5$. One can see that the density of zero momentum
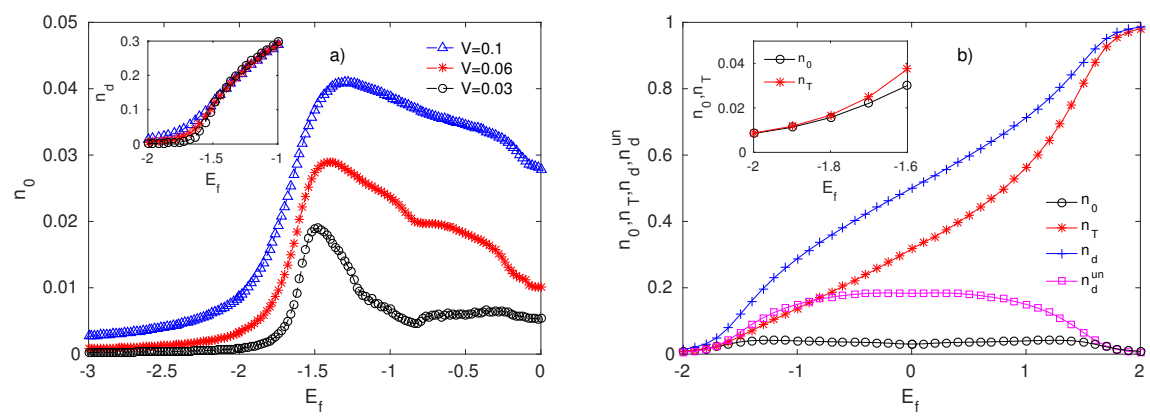

Figure 4. (Colour online) a) $n_{0}$ as a function of $E_{f}$ calculated for three different values of $V\left(t_{f}=\right.$ $0, U=0.5, L=\infty)$. The inset shows the density of $d$ electrons $n_{d}$ near $E_{f}=-1.5$. b) $n_{0}, n_{T}, n_{d}$ and $n_{d}^{\text {un }}=n_{d}-n_{T}$ as functions of $E_{f}$ calculated for $t_{f}=0, U=0.5, V=0.1$ and $L=\infty$. The inset shows the behaviour of $n_{0}$ and $n_{T}$ near $E_{f}=-2$ [37].

excitons is nonzero over the whole interval of $E_{f}$ values. Moreover, we have found that the values of $n_{0}$ are extremely enhanced in the region near $E_{f} \sim-1.5$, which is obviously due to a significant enhancement of the $d$ electron population in the $d$ band (see the inset in figure $4 \mathrm{a}$ ).

To describe the process of formation of excitonic bound states with increasing $E_{f}$ more in detail, we have also plotted in figure $4 \mathrm{~b}$, besides the density of zero momentum excitons $n_{0}$, the total exciton density $n_{T}$, the total $d$-electron density $n_{d}$ and the total density of unbond $d$ electrons $n_{d}^{\text {un }}=n_{d}-n_{T}$. It is seen (see the inset in figure $4 \mathrm{~b}$ ) that below $E_{f} \sim-1.8, n_{0}$ and $n_{T}$ coincides, which means that the excitonic insulator in this region is practically completely driven by the condensation of zero-momentum excitons. Above this value $n_{T}$ starts to sharply increase, while $n_{0}$ tends to its maximum at $E_{f} \sim-1.3$ and then gradually decreases to its minimum at $E_{f}=0$. Similar behaviour with increasing $E_{f}$ also exhibits the density of unbond $d$ electrons $n_{d}^{\text {un }}$, though the values of $n_{d}^{\text {un }}$ are several times larger than $n_{0}$. It is interesting to note that although the total exciton density $n_{T}$ increases over the whole interval of $E_{f}$ values, the number of unbond $d$ electrons remains practically unchanged over the wide range of $E_{f}$ values (from $E_{f}=-1$ to $E_{f}=1$ ), since its decrease, due to the formation of excitonic pairs, is compensated by the increase of $n_{d}\left(E_{f}\right)$. Thus, we can conclude that in the pressure induced case, when the $f$-level energy shifts up with the applied pressure [42], the model is capable of describing, at least qualitatively, the increase in the total density of excitons with external pressure and the increase or decrease (according to the initial position of $E_{f}$ at ambient pressure) in the $n_{0}$ and $n_{d}^{\text {un }}$.

\subsection{Effects of non-local hybridization with inversion symmetry}

As already mentioned, from the physics viewpoint, the most interesting case corresponds to the case of finite non-local hybridization [37]. The importance of this term emphasizes the fact that the on-site hybridization $V$ is usually forbidden in real $d-f$ systems for parity reasons. Instead of the onsite hybridization, one should consider in these materials the non-local hybridization with inversion symmetry $V_{i, j}=V_{\text {non }}\left(\delta_{j, i-1}-\delta_{j, i+1}\right)$ which leads to $k$-dependent hybridization of the opposite parity that corresponds to the $d$ band [ $V_{k} \sim \sin (k)$ ] [43]. Typical examples of $1 / L$ dependence of the excitonic momentum distribution $N(q=0)$ obtained for three representative values of the interband Coulomb interaction and two values of $f$-electron hopping are displayed in figure $5 \mathrm{a}$ and figure $5 \mathrm{~b}$. These results clearly demonstrate that there is no sign of divergence in the $1 / L$-dependence of $N(0)$ neither for $t_{f}=0$ nor for $t_{f}=-0.05$ and thus, there is no signal of forming the Bose-Einstein condensate in the presence of non-local hybridization with the inversion symmetry. Thus, our results indicate that the class of possible candidates for the appearance of the Bose-Einstein condensation of excitons in real $d-f$ materials is strongly limited, since the local hybridization is usually forbidden in these systems for parity 

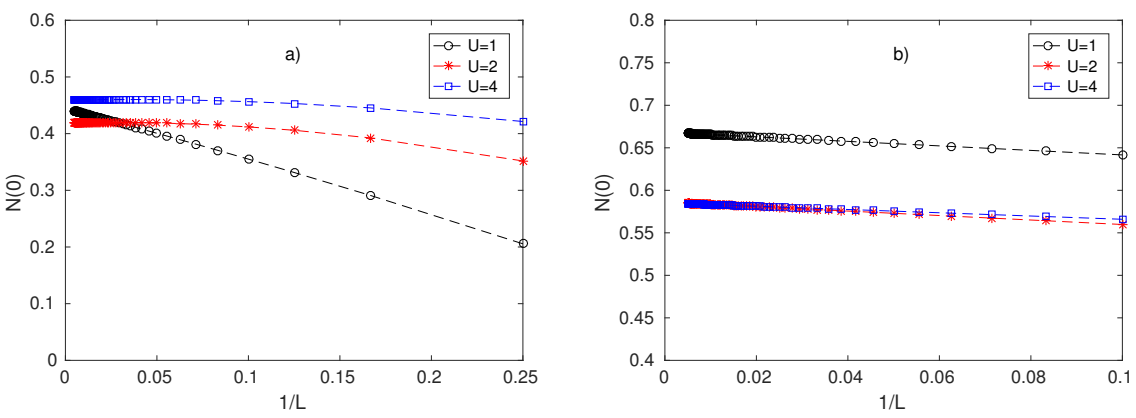

Figure 5. (Colour online) $N(0)$ as a function of $1 / L$ calculated for three different values of $U$ and two different values of $t_{f}$ : a) $\left.t_{f}=0, \mathrm{~b}\right) t_{f}=-0.5\left(E_{f}=0, V_{\text {non }}=0.1\right)$ [37].

reasons and the non-local hybridization with the inversion symmetry does not support the formation of the Bose-Einstein condensate.

\subsection{Combined effects of local and non-local hybridization with equal parity of $\boldsymbol{d}$ and $f$ orbitals}

In this situation, the most promising candidates for studying this phenomenon seem to be the systems with equal parity of $d$ and $f$ orbitals, where the nonlocal hybridization $H_{\mathrm{n}}$ can be written as [38]:

$$
H_{\mathrm{n}}=V_{\mathrm{n}} \sum_{\langle i, j\rangle}\left(d_{i}^{+} f_{j}+H . c .\right) \text {. }
$$

In such systems, the local hybridization $V$ is allowed, and thus one can examine the combined effects of the local and nonlocal hybridization within the unified picture. In the weak $(U<1)$ and strong $(V \ll U$ and $V_{\mathrm{n}} \ll U$ ) coupling limits, the model Hamiltonian $H_{0}+H_{V}+H_{\mathrm{n}}$ was recently analyzed by Zenker et al. in [44], and the corresponding mean-field quantum phase diagrams were presented as functions of the model parameters $U, V, V_{\mathrm{n}}$ and $E_{f}$ for the half-filed band case $n_{f}+n_{d}=1$ and $D=2$. Moreover, examining the effects of the local $V$ and nonlocal $V_{\mathrm{n}}$ hybridization, they found that in the pseudospin space $\left(c_{i \uparrow}^{+}=d_{i}^{+}, c_{i \downarrow}^{+}=f_{i}^{+}\right)$, the nonlocal hybridization $V_{\mathrm{n}}$ favors the staggered Ising-type ordering along the $x$ direction, while $V$ favors a uniform polarization along the $x$ direction and the staggered Ising-type ordering along the $y$ direction. In our paper [38] we have examined the model for arbitrary $V$ and $V_{\mathrm{n}}$ and unlike the paper of Zenker et al. [44] we have focused our attention primarily on a description of process of formation and condensation of exitonic bound states.

Let us discuss the results obtained for $n_{0}=\frac{1}{L} N(q=0), n_{\pi}=\frac{1}{L} N(q=\pi), n_{d}$ and $n_{d}^{\text {un }}$ as functions of the $f$-level position $E_{f}$ which can give us, at least qualitatively, the answer to the very important question, and namely, how these quantities change with the applied pressure $p$. In figure 6 we present the resultant behaviours of $n_{0}, n_{\pi}, n_{d}, n_{d}^{\text {un }}$ as functions of the $f$-level position $E_{f}$ obtained by the DMRG method for $V=0.2$ and several different values of $V_{\mathrm{n}}$. In all examined cases, the density of zero-momentum excitons is the most significantly enhanced for $d$-electron densities near the half-filled band case $E_{f}=0$ and $n_{d}=1 / 2$. The changes of $n_{0}$ are gradual for $E_{f}<0$ and very steep, but still continuous, for $E_{f}>0$. The fully different behaviour exhibits the density of $\pi$-momentum excitons $n_{\pi}$. Its enhancement with increasing $E_{f}$ is practically negligible for $E_{f}<0$, but from this value $n_{\pi}$ it starts to sharply increase and tends to its saturation value corresponding to the fully occupied $d$ band $n_{d} \sim 1$. The density of unbound $d$ electrons $n_{d}^{\text {un }}$ exhibits a very simple behaviour for $E_{f}<0$. In this limit, $n_{d}^{\text {un }}$ gradually increases with increasing $E_{f}$ for all examined values of nonlocal hybridization $V_{\mathrm{n}}$. However, in the opposite case $\left(E_{f}>0\right)$, the density of unbound $d$ electrons $n_{d}^{\text {un }}$ behaves fully differently for $V_{\mathrm{n}}<V_{n}^{c}$ and $V_{\mathrm{n}}>V_{n}^{c}$, where $V_{n}^{c} \sim 0.2$. For $V_{\mathrm{n}}<V_{n}^{c}$, the density of unbound $d$ electrons $n_{d}^{\text {un }}$ gradually decreases with an increasing $E_{f}$ and tends to zero when $E_{f}$ approaches the upper edge of the noninteracting band $E_{f}=2$, but in the opposite limit the density of unbound $d$ electrons $n_{d}^{\text {un }}$ decreases by the interval of $E_{f}$ values from $E_{f}=0$ 

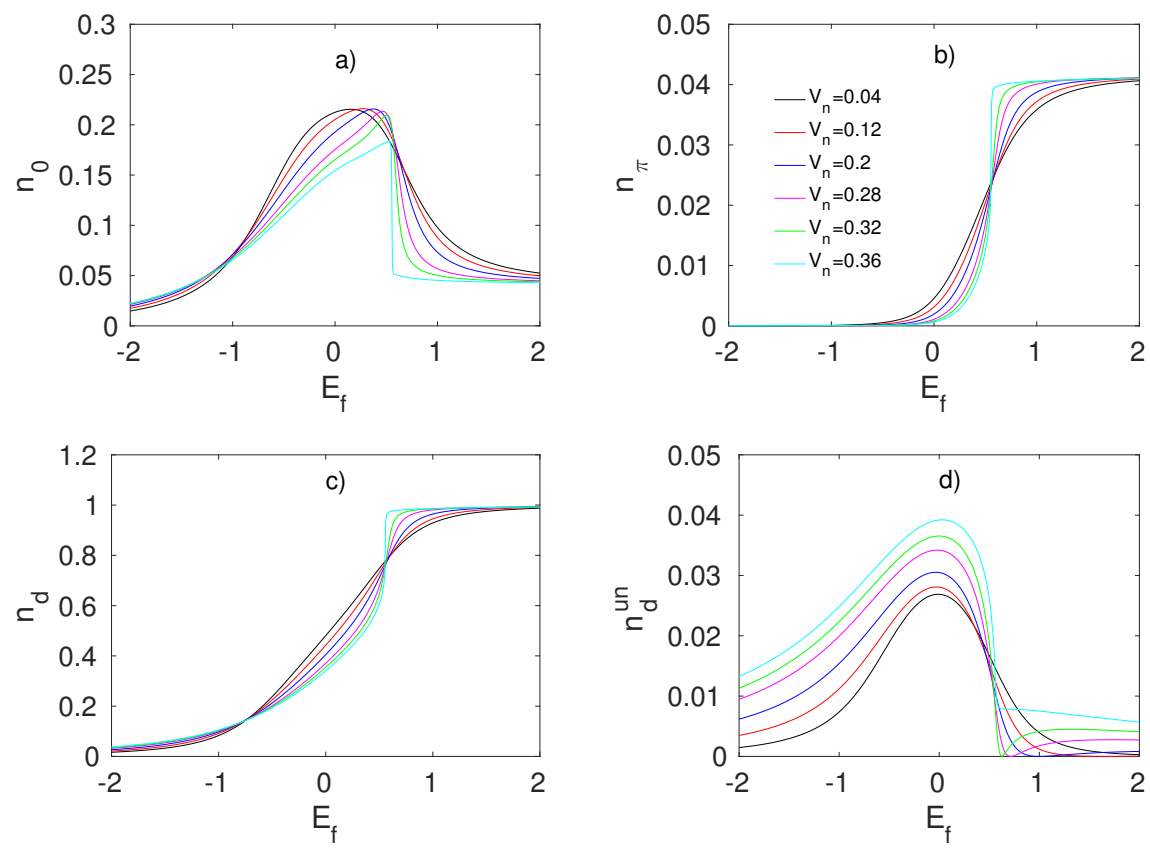

Figure 6. (Colour online) The density of zero-momentum excitons $n_{0}$ (a), the density of $\pi$-momentum excitons $n_{\pi}$ (b), the total $d$-electron density $n_{d}$ (c), and the total density of unbound $d$ electrons $n_{d}^{\text {un }}=$ $n_{d}-n_{T}(\mathrm{~d})$ as functions of $E_{f}$ calculated for $U=4, V=0.2, L=60$ and six different values of $V_{\mathrm{n}}$ [38].

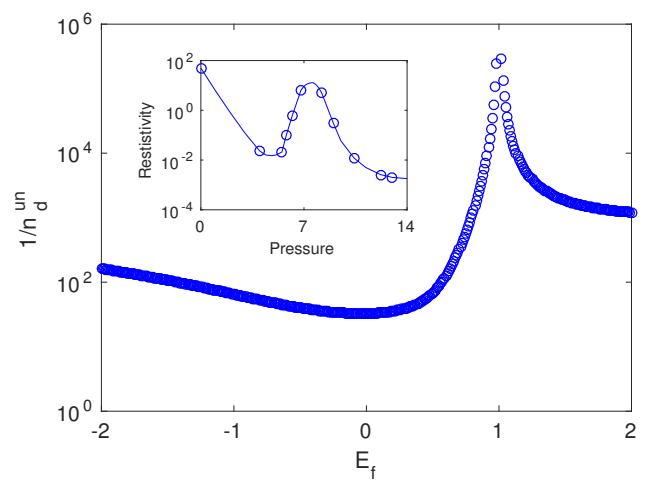

Figure 7. (Colour online) The inverse value of the density of unbound $d$-electrons $n_{d}^{\text {un }}$ as a function of the $f$-level energy $E_{f}$ calculated for $U=4, V=0.2, V_{\mathrm{n}}=0.2$ and $L=\infty$ [38]. The inset shows the resistivity as the function of pressure in $\mathrm{TmSe}_{0.45} \mathrm{Te}_{0.55}$ at $4.2 \mathrm{~K}$ [6].

to $E_{f}^{c}\left(V_{\mathrm{n}}\right)$, and $n_{d}^{\text {un }}$ starts to increases again for $E_{f}>E_{f}^{c}\left(V_{\mathrm{n}}\right)$. Taking into account the above mentioned parametrization between $E_{f}$ and the external pressure $p$, as well as the fact that the electrical conductivity is proportional to the density of unbound electrons $n_{d}^{\text {un }}$ (and the electrical resistivity to $1 / n_{d}^{\text {un }}$ ), the results discussed above could have very important physical consequences. Indeed, in figure 7 we have plotted the quantity $1 / n_{d}^{\text {un }}$ (in the logarithmic scale) as a function of $E_{f}$ and compare it with experimental measurements of the pressure dependence of the electrical resistivity in the mixed valence compound $\mathrm{TmSe}_{0.45} \mathrm{Te}_{0.55}$ (see the inset in figure 7). One can see that there is a nice qualitative accordance between our theoretical predictions and experimental results of Wachter et al. [6]. In spite of the fact that our model is in many aspects very simplified, the physics that could lead to the unusual behaviour of the 
electrical resistivity in $\mathrm{TmSe}_{0.45} \mathrm{Te}_{0.55}$ under the external pressure seems to be clear. This is a result of the formation and condensation of excitonic bound states of conduction-band electrons and valence-band holes.

\subsection{Effects of non-local Coulomb interactions}

The above discussed results show that the Falicov-Kimball model has a great potential to describe some of the anomalous features of real complex materials such as rare-earth compounds. On the other hand, it should be noted that the original version of the model, as well as its extensions discussed above, represent a too crude approximation of real rare-earth compounds, since we neglect all nonlocal Coulomb interactions, that can change this picture. For a correct description of these materials one should take into account at least the following nonlocal Coulomb interaction terms [39]:

$$
H_{\mathrm{non}}=U_{d d} \sum_{<i j>} n_{i}^{d} n_{j}^{d}+U_{d f} \sum_{<i j>} n_{i}^{d} n_{j}^{f}+U_{f f} \sum_{<i j>} n_{i}^{f} n_{j}^{f}+U_{c h} \sum_{<i j>} d_{i}^{+} d_{j}\left(n_{i}^{f}+n_{j}^{f}\right)
$$

which represent the nearest-neighbour Coulomb interaction between two $d$ electrons (the first term), between one $d$ and one $f$ electron (the second term), between two $f$ electrons (the third term) and the so-called correlated hopping (the last term).

There is a number of papers, were the influence of individual interaction terms from (2.2) on the ground state properties of the Falicov-Kimball model has been studied. However, there are only a few where the combined effects of two or three terms were considered. Among the papers dealing with the influence of individual interactions, let us mention the work [45] (and references therein) where the effects of nonlocal interaction between $d$ and $f$ electrons are examined and the excellent papers of Shvaika et al. [46-48] where rigorous results for the influence of the correlated hopping on the thermodynamical functions were derived within the local approach and then used for a description of various physical problems. Among the papers dealing with combined effects of two or three terms, let us mention the works [49, 50] (and references therein). From this point of view, the model Hamiltonian $H=H_{0}+H_{V}+H_{\text {non }}$ considered here represents one of the most complex extensions of the FalicovKimball model used for a description of ground state properties of strongly correlated systems. Here, we focus our attention exclusively on a discussion of two main problems, and namely, the process of formation and condensation of excitonic bound states and the problem of valence transitions in the generalized Falicov-Kimball model. To simplify numerical calculations, we adopt here the following model $U_{d d}=U_{f f}=U_{d f}=U_{n n}$, that allows us to reduce the number of model parameters and at the same time to keep all nonlocal interaction terms nonzero. The physically most interesting case corresponds to the situation where both $\left(U_{n n}\right.$ as well as $\left.U_{c h}\right)$ interactions are switched on simultaneously and numerical results for this case are summarized in figure 8 . One can see that combined effects of nonlocal interactions lead to a number of interesting results: (i) strong suppression of the zero-momentum condensate in the region of $E_{f}$, where $n_{d} \sim 0.5$, (ii) stabilization of the intermediate phase with $n_{d} \sim 0.5$ for increasing $U_{n n}=U_{c h}$, (iii) strong enhancement of the total density of unbond $d$ electrons $n_{d}^{\text {un }}$ with an increase of $U_{n n}=U_{c h}$. (iv) stabilization of zero momentum condensate for some values of the $f$ level energy $E_{f}$ in the weak coupling limit $U_{n n}=U_{c h} \sim 0.2$, (v) appearance of discontinuous valence transitions for sufficiently large values of $U_{n n}=U_{c h} \sim 0.4$ and (vi) discontinuous disappearance of the density of zero momentum excitons, as well as discontinuous changes in the total density of excitons $n_{T}$ and the total density of unbond $d$ electrons $n_{d}^{\text {un }}$ at the valence transition points.

The appearance of discontinuous changes in some ground-state observables such as the density of conduction $d$ (valence $f$ ) electrons, the density of zero-momentum condensate, the density of unbond electrons, is a very important result from the point of view of rare-earth compounds. In some of them, e.g., the mixed valence system $\mathrm{SmS}$ such discontinuous changes are experimentally observed in the density of valence electrons when the external hydrostatic pressure is applied [51], though they were not satisfactorily described so far. Indeed, as mentioned above, the SmS compound is a mixed valence system, with fluctuating valence and thus for its description one should take into account the hybridization between the localized $f$ and conduction $d$ electron states. However, more reliable methods, such as alloy-analog approximation [52], renormalization group method [53], exact diagonalization method [54], predict only 

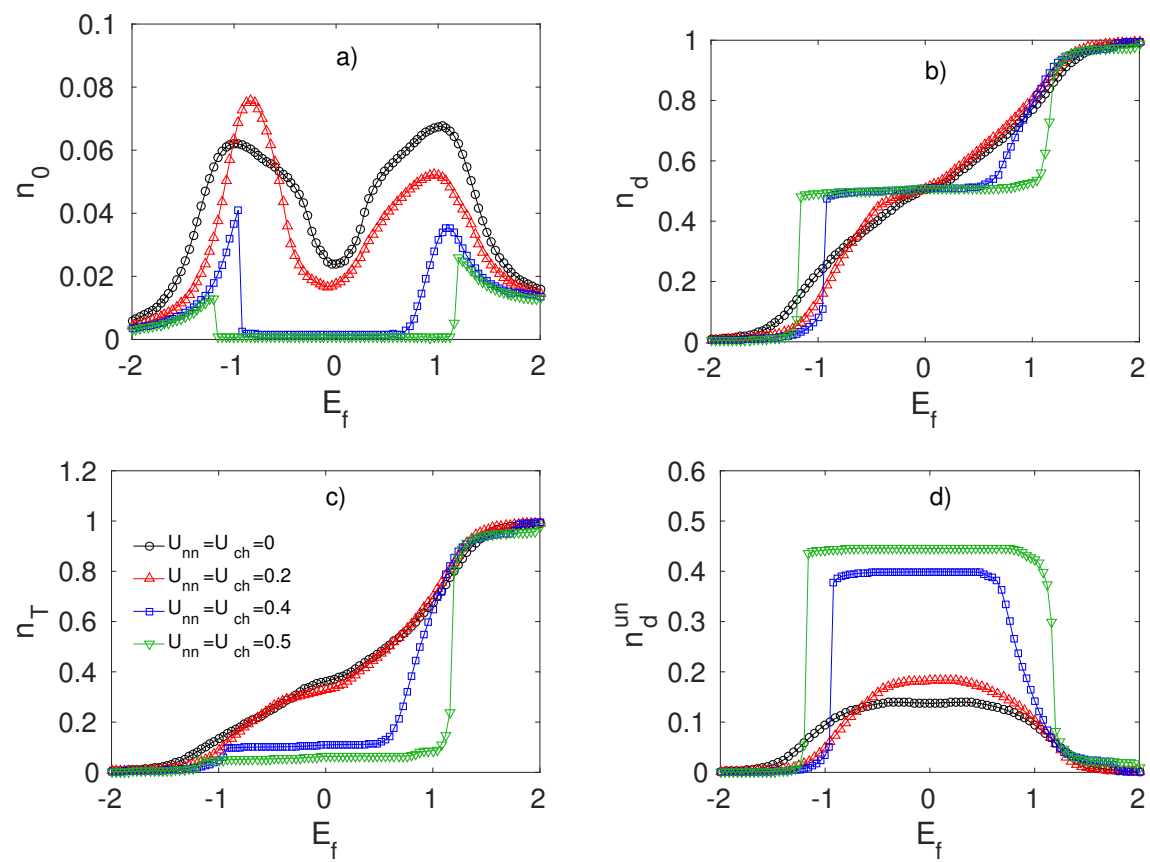

Figure 8. (Colour online) $n_{0}, n_{d}, n_{T}$ and $n_{d}^{\text {un }}=n_{d}-n_{T}$ as functions of $E_{f}$ calculated for four different values of $U_{c h}\left(U_{c h}=0,0.2,0.4,0.5\right)$ at $U_{n n}=U_{c h}, U=1, V=0.1, L=100$ and $n_{f}+n_{d}=1$ [39].

the continuous valence transitions within the Falicov-Kimball model extended by the local hybridization. Here, we show that considering the parametrization between the external pressure $p$ and the $f$-level position $E_{f}$, the pressure induced discontinuous valence transitions are possible to generate also in such a system under a very realistic assumption, namely, that nonlocal interactions are switched on. This opens up a new route to the understanding of various ground-state anomalies observed in the rare-earth compounds within the unified picture.

Finally, it should be noted that although all the results presented in this review were obtained for the one-dimensional case, their validity is probably much more general. Indeed, a direct comparison of our one-dimensional DMRG and two dimensional Hartree-Fock results [37], obtained for the density of zero-momentum excitons as a function of $t_{f}$ and $E_{f}$, revealed only a weak dependence of $n_{0}$ on the system dimension indicating a possible extension of our one-dimensional DMRG results to real two and three dimensional systems. Moreover, in the two-dimensional case, we can switch off completely the local hybridization, since in this case the excitonic condensate can be generated by other terms (the $f$-electron hopping), modelling more realistically the situation in rare-earth compounds.

\section{Acknowledgements}

This work was supported by projects VEGA 2-0112-18, APVV-17-0020, ITMS 2220120047, ITMS 26230120002 and IMTS 26210120002.

\section{References}

1. Blatt J.M., Böer K.W., Brandt W., Phys. Rev., 1962, 126, 1691, doi 10.1103/PhysRev.126.1691

2. Keldysh L.V., Kopaev H.Y.V., Sov. Phys. Solid State, 1965, 6, 2219.

3. Moskalenko S.A., Snoke D.W., Bose-Einstein Condensation of Excitons and Biexcitons, Cambridge Univ. Press, Cambridge, 2000. 
4. Littlewood P.B., Eastham P.R., Keeling J.M.J., Marchetti F.M., Simons D.B., Szymanska M.H., J. Phys.: Condens. Matter, 2004, 16, S3597, doi $10.1088 / 0953-8984 / 16 / 35 / 003$

5. Des Cloizeaux J., J. Phys. Chem. Solids, 1965, 26, 259, doi 10.1016/0022-3697(65)90153-8.

6. Neuenschwander J., Wachter P., Phys. Rev. B, 1990, 41, 12693, doi:10.1103/PhysRevB.41.12693.

7. Bucher B., Steiner P., Wachter P., Phys. Rev. Lett., 1991, 67, 2717, doi:10.1103/PhysRevLett.67.2717

8. Monney C., Monney G., Aebi P., Beck H., Phys. Rev. B, 2012, 85, 235150, doi: $10.1103 /$ PhysRevB.85.235150

9. Zenker B., Fehske H., Beck H., Monney C., Bishop A.R., Phys. Rev. B, 2013, 88, 075138, doi 10.1103/PhysRevB.88.075138.

10. Monney G., Monney C., Hildebrand B., Aebi P., Beck H., Phys. Rev. Lett., 2015, 114, 086402, doi $10.1103 /$ PhysRevLett.114.086402

11. Watanabe H., Seki K., Yunoki S., Phys. Rev. B, 2016, 91, 205135, doi 10.1103/PhysRevB.91.205135

12. Wakisaka I., Sudayama T., Takubo K., Mizokawa T., Arita M., Namatame H., Taniguchi M., Katayama N., Nohara M., Takagi H., Phys. Rev. Lett., 2009, 103, 026402 doi 10.1103/PhysRevLett.103.026402.

13. Perali A., Neilson D., Hamilton A.R., Phys. Rev. Lett., 2013, 110, 146803, doi:10.1103/PhysRevLett.110.146803

14. Falicov L.M., Kimball J.C., Phys. Rev. Lett., 1969, 22, 997, doi 10.1103/PhysRevLett.22.997.

15. Portengen T., Östreich T., Sham L.J., Phys. Rev. Lett., 1996, 76, 3384, doi 10.1103/PhysRevLett.76.3384.

16. Portengen T., Östreich T., Sham L.J., Phys. Rev. B, 1996, 54, 17452, doi:10.1103/PhysRevB.54.17452

17. Czycholl G., Phys. Rev. B, 1999, 59, 2642, doi:10.1103/PhysRevB.59.2642

18. Farkašovský P., Phys. Rev. B, 1999, 59, 9707, doi 10.1103/PhysRevB.59.9707.

19. Farkašovský P., Phys. Rev. B, 2002, 65, 81102, doi 10.1103/PhysRevB.65.081102.

20. Zlatić V., Freericks J.K., Lemanski R., Czycholl G., Philos. Mag. B, 2001, 81, 1443, doi $10.1080 / 13642810110066470$.

21. Freericks J.K., Zlatić V., Rev. Mod. Phys., 2003, 75, 1333, doi 10.1103/RevModPhys.75.1333

22. Batista C.D., Phys. Rev. Lett., 2002, 89, 166403, doi 10.1103/PhysRevLett.89.166403

23. Batista C.D., Gubernatis J.E., Bonča, Lin H.Q., Phys. Rev. Lett., 2004, 92, 187601, doi 10.1103/PhysRevLett.92.187601.

24. Farkašovský P., Phys. Rev. B, 2008, 77, 155130, doi:10.1103/PhysRevB.77.155130

25. Schneider C., Czycholl, Eur. Phys. J. B, 2008, 64, 43, doi $10.1140 /$ epjb/e2008-00273-y.

26. Zenker B., Ihle D., Bronold F.X., Fehske H., Phys. Rev. B, 2010, 81, 115122, doi:10.1103/PhysRevB.81.115122

27. Phan V.N., Becker K.W., Fehske H., Phys. Rev. B, 2010, 81, 205117, doi 10.1103/PhysRevB.81.205117

28. Seki K., Eder R., Ohta Y., Phys. Rev. B, 2011, 84, 245106, doi 10.1103/PhysRevB.84.245106

29. Zenker B., Ihle D., Bronold F.X., Fehske H., Phys. Rev. B, 2012, 85, 121102R, doi $10.1103 /$ PhysRevB.85.121102.

30. Kaneko T., Seki K., Ohta Y., Phys. Rev. B, 2012, 85, 165135, doi 10.1103/PhysRevB.85.165135

31. Kaneko T., Ejima S., Fehske H., Ohta Y., Phys. Rev. B, 2013, 88, 035312, doi:10.1103/PhysRevB.88.035312

32. Ejima S., Kaneko T., Ohta Y., Fehske H., Phys. Rev. Lett., 2014, 112, 026401, doi $10.1103 /$ PhysRevLett.112.026401

33. Apinyan V., Kopeć T.K., J. Low Temp. Phys., 2014, 176, 27, doi:10.1007/s10909-014-1165-x

34. Kuneš J., J. Phys.: Condens. Matter, 2015, 27, 333201, doi /10.1088/0953-8984/27/33/333201

35. Golosov D.I., Phys. Rev., 2020, 101, 165130, doi:10.1103/PhysRevB.101.165130

36. Farkašovský P., EPL, 2015 110, 47007 doi $10.1209 / 0295-5075 / 110 / 47007$

37. Farkašovský P., Phys. Rev. B, 2017 95, 045101, doi 10.1103/PhysRevB.95.045101

38. Farkašovský P., Solid State Commun., 2017, 255, 24, doi $10.1016 /$ j.ssc.2017.03.005

39. Farkašovský P., Regeciová L., Eur. Phys. J. B, 2019, 92, 141, doi 10.1140/epjb/e2019-90406-6.

40. White S.R., Phys. Rev. Lett., 1992, 69, 2863, doi:10.1103/PhysRevLett.69.2863

41. Brouers F., de Menezes O.L.T., Phys. Status Solidi B, 1981, 104, 541, doi $10.1002 /$ pssb.2221040218

42. Gonçalves da Silva C.E.T., Falicov L.M., Solid State Commun., 1975, 17, 1521, doi:10.1016/00381098(75)90986-2.

43. Czycholl G., Phys. Rep. B, 1986, 143, 277, doi 10.1016/0370-1573(86)90177-8

44. Zenker B., Fehske H., Batista C.D., Phys. Rev. B, 2010, 82, 165110, doi $10.1103 /$ PhysRevB.82.165110.

45. Farkašovský P., Acta Phys. Slovaca, 2010, 60, 497, doi:10.2478/v10155-010-0005-z

46. Shvaika A.M., Phys. Rev. B, 2003, 67, 075101, doi 10.1103/PhysRevB.67.075101

47. Shvaika A.M., Condens. Matter Phys., 2014, 17, 43704, doi:10.5488/CMP.17.43704

48. Dobushovskyi D.A., Shvaika A.M., Zlatić V., Phys. Rev. B, 2017, 95, 125133, doi:10.1103/PhysRevB.95.125133

49. Čenčariková H., Farkašovský P., Žonda M., Acta Phys. Pol. A, 2008, 113, 287, doi 10.12693/APhysPolA.113.287

50. Lemański R., Kapcia K.J., Robaszkiewicz S., Phys. Rev. B, 2017, 96, 205102, doi 10.1103/PhysRevB.96.205102

51. Rohler J., Handbook on the Physics and Chemistry of Rare Earths, Vol. 10, Gschneider K.A., Eyring L.R., 
Huffner S. (Eds.) Amsterdam: North-Holland, 453.

52. Czycholl G., Phys. Rep., 1986, 143, 277-345, doi 10.1016/0370-1573(86)90177-8

53. Hanke W., Hirsch J.E., Phys. Rev. B, 1982, 25, 6748, doi $10.1103 /$ PhysRevB.25.6748

54. Farkašovský P., Z. Phys. B, 1997, 104, 553, doi 10.1007/s002570050489

\section{Дослідження конденсації екситонів методом DMRG для узагальненої моделі Фалікова-Кімбала}

\section{П. Фаркашовський}

Інститут експериментальної фізики, Словацька академія наук, вул. Ватсонова 47, Кошиці, Словаччина

Формування і конденсація зв'язаних екситонних станів між електронами з зони провідності та дірками з валентної зони, безумовно, належить до однієї з найбільш захоплюючих ідей сучасної фізики твердого тіла. У цьому короткому огляді, ми представляємо останній прогрес у цій галузі, що був досягнутий завдяки розрахункам методом ренорм групи матриці густини (DMRG) для різних узагальнень моделі ФаліковаКімбала. Особлива увага приділяється опису найважливіших механізмів (взаємодій), які впливають на стабільність екситонної фази, а саме: (i) міжзонна $d$ - $f$ кулонівська взаємодія, (iі) перенос $f$-електронів, (iii) парна і непарна нелокальна гібридизація, (iv) комбіновані ефекти локальної та нелокальної гібридизації, (v) кулонівська взаємодія найближчих сусідів між $d$ - і $f$-електронами та (vi) корельований перенос. Широко обговорюється відповідність числових результатів, отриманих для різних узагальнень моделі Фалікова-Кімбала, для опису реальних $d-f$ матеріалів.

Ключові слова: модель Фалікова-Кімбалла, квантові конденсати, одновимірні системи 


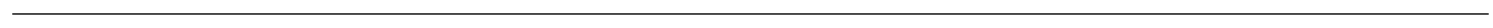

